\title{
AN OPTIMAL SLIDING MODE CONGESTION CONTROLLER FOR CONNECTION-ORIENTED COMMUNICATION NETWORKS WITH LOSSY LINKS
}

\author{
ANDRZEJ BARTOSZEWICZ, PIOTR LEŚNIEWSKI \\ Institute of Automatic Control \\ Technical University of Łódź, Bohdana Stefanowskiego 18/22, 90-924 Łódź, Poland \\ e-mail: andrzej .bartoszewicz@p.lodz.pl, piotr.lesniewski2@gmail.com
}

\begin{abstract}
A new discrete-time sliding-mode congestion controller for connection-oriented networks is proposed. Packet losses which may occur during the transmission process are explicitly taken into account. Two control laws are presented, each obtained by minimizing a different cost functional. The first one concentrates on the output variable, whereas in the second one the whole state vector is considered. Weighting factors for adjusting the influence of the control signal and appropriate (state or output) errors are incorporated in both the functionals. The asymptotic stability of the closed-loop system is proved, and the conditions for $100 \%$ bottleneck node bandwidth utilization are derived. The performance of the proposed algorithm is verified by computer simulations.
\end{abstract}

Keywords: optimal control, sliding-mode control, flow control, discrete-time systems.

\section{Introduction}

In connection-oriented communication networks, data units sent by sources pass through a series of intermediate nodes before reaching their destinations. If an intermediate node due to a limited data flow rate of its outgoing link cannot pass on all the data it receives, then congestion occurs. Consequently, in order to maximize throughput as well as minimize queuing delays and jitter in modern communication networks, congestion control algorithms are applied. The main difficulty in appropriate congestion control algorithm design is caused by large propagation delays in the networks. The delays are inevitable since information about congestion at a specific node must be dispatched to all sources transmitting data through this node, in order to enable adjustment of the source transmission rates, and this action does not affect the congested node immediately, but only with delay usually called the Round Trip Time (RTT). The problem of congestion control in connection-oriented communication networks has been studied in many papers, and an extensive review of the papers can be found in a recent monograph of Ignaciuk and Bartoszewicz (2013).

The main advantage of sliding mode control methods is their strong robustness with regard to a class of disturbance and model uncertainty (Drazenovic, 1969).
The robustness is achieved by employing nonlinear control signals to force the system trajectory to attain in finite time a motion along a pre-determined surface in the state space. The sliding mode approach was originally proposed for controller design (Emelyanov, 1967; Gao et al., 1995; Hung et al., 1993; Levant, 1993; Mnasri and Gasmi, 2011; Sira-Ramirez, 1993; Tomera, 2010; Utkin, 1977; Utkin and Shi, 1996). However, sliding mode techniques can also be used in state observers (Edwards and Spurgeon, 1994; Edwards et al., 2012; Floquet et al., 2007; Fridman et al., 2007; Haskara et al., 1998; Veluvolu and Soh, 2009).

Due to the robustness of sliding mode control, various types of sliding mode congestion controllers have been proposed. Jing et al. (2007) used a sliding mode controller with a state predictor, and established the maximum delay necessary for the system stability. A fuzzy controller combining the advantages of linear and terminal sliding modes was also proposed by Jing et al. (2008) for a simplified delay-free network model. For a DiffServ network, an adaptive sliding mode controller (using the backstepping procedure) for a model which neglects the feedback latency was presented by Zheng et al. (2008). On the other hand, for a DiffServ network with delay a second order sliding mode technique was applied by Zhang 
et al. (2009) in order to reduce the chattering of the control signal. In the work of Jin et al. (2009) the problem of fair (in the max-min sense) data rate distribution among the sources is considered. A binary congestion signal is used to control the data output of sources, and the analysis of this algorithm is performed for a delay-free system.

The papers mentioned in the previous paragraph use the continuous time network model. However, any flow control algorithm for a data transmission network must be implemented as a digital controller. Therefore, in some works a discrete-time approach to the problem of data flow control was used. For example, a sliding mode controller was presented by Yan et al. (2007), but the result of that paper was derived without considering the system delays. In the work of Yang et al. (2007) it is shown that any max-min fair system with a stable symmetric Jacobian matrix maintains asymptotic stability under arbitrary directional delays. This means that, if the controller is designed so that the system has a symmetric Jacobian matrix, its stability can be examined based on the corresponding undelayed system. A dead-beat sliding mode controller for multi-source networks with a priori known round trip times is presented by Bartoszewicz and Zuk (2009), whereas in the work of Ignaciuk and Bartoszewicz (2008) an LQ optimal sliding mode controller for single-source networks is proposed. The same approach is then extended for multi-source networks by Ignaciuk and Bartoszewicz (2009), who also design a similar optimal flow controller for multi-source networks with the round trip times which may change during the control process (Ignaciuk and Bartoszewicz, 2011).

In most papers published up to now only packet losses due to buffer overflows are considered, and the occurrence of lossy links in the network is neglected. As in real networks transmission losses are inevitable, in this paper we present an LQ optimal sliding mode controller for a single connection in which packets may be lost during the transmission process.

\section{Network model}

In this paper we consider a single virtual circuit in a connection-oriented network. The virtual circuit consists of a single data source, intermediate nodes and a destination. Data sent by the source are passed from node to node, until they reach their destination. It is assumed that one of the intermediate nodes (further in the paper called the "bottleneck" node) cannot pass on all the data it receives, due to the limited bandwidth of its outgoing link. Thus congestion occurs, and the surplus data accumulate in the buffer located at the bottleneck node. The block diagram of the network is shown in Fig. 1

We assume that the source is persistent, i.e., it always has enough data to transmit at the maximum rate allowed by the network. Therefore, the congestion problem can be solved through an appropriate adjustment of the data rate of the source. This rate is determined by the controller placed at the bottleneck node. The source receives the signal from the controller (denoted by $u$ ) after backward delay $T_{B}$. It then sends the specified amount of data, which reaches the bottleneck queue after forward delay $T_{F}$. It is assumed that during the transmission some data packets are lost, so that only $\alpha u(\alpha \in(0,1))$ data packets arrive at the bottleneck node. The round trip time RTT (the delay between generating the control signal, and the requested data arriving at the bottleneck queue) can be expressed as a sum of backward and forward propagation delays,

$$
\mathrm{RTT}=T_{B}+T_{F} .
$$

Further in the paper, $T$ represents the sampling time. The queue length at time instant $k T$ is denoted by $y(k T)$, and its demand value by $y_{d}$. It is assumed that before the beginning of data transmission the buffer is empty, i.e., $y(k T<0)=0$. The controller output at time $k T$ is denoted by $u(k T)$. We also assume that the round trip time is a multiple of the sampling time, i.e., $\mathrm{RTT}=m_{\mathrm{RTT}} T$, where $m_{\mathrm{RTT}}$ is a positive integer.

The amount of data which may leave the bottleneck buffer at each time instant is modeled as an a-priori unknown bounded function of time $d(k T)$, and its maximum value is denoted by $d_{\max }$. Because at some time instants there can be less data in the queue than can be sent, an additional function of time $h(k T)$ is introduced, which represents the data actually leaving the bottleneck queue. Consequently,

$$
0 \leq h(k T) \leq d(k T) \leq d_{\max }
$$

The queue length for any $k T \geq 0$ can be expressed as follows:

$$
\begin{aligned}
y(k T) & =\alpha \sum_{j=0}^{k-1} u(j T-R T T)-\sum_{j=0}^{k-1} h(j T) \\
& =\alpha \sum_{j=0}^{k-m_{\mathrm{RTT}}-1} u(j T)-\sum_{j=0}^{k-1} h(j T) .
\end{aligned}
$$

The network can also be described in the state space as

$$
\begin{aligned}
\boldsymbol{x}[(k+1) T] & =\boldsymbol{A x}(k T)+\boldsymbol{b} u(k T)+\boldsymbol{o} h(k T), \\
y(k T) & =\boldsymbol{q}^{T} \boldsymbol{x}(k T)
\end{aligned}
$$

where $\boldsymbol{x}(k T)=\left[\begin{array}{llll}x_{1}(k T) & x_{2}(k T) & \cdots & x_{n}(k T)\end{array}\right]^{T}$ is the state vector, $\operatorname{dim}(\boldsymbol{x})=n=m_{\mathrm{RTT}}+1, y(k T)=x_{1}(k T)$ is the queue length, and

$$
x_{i}(k T)=u[(k-n+i-1) T]
$$




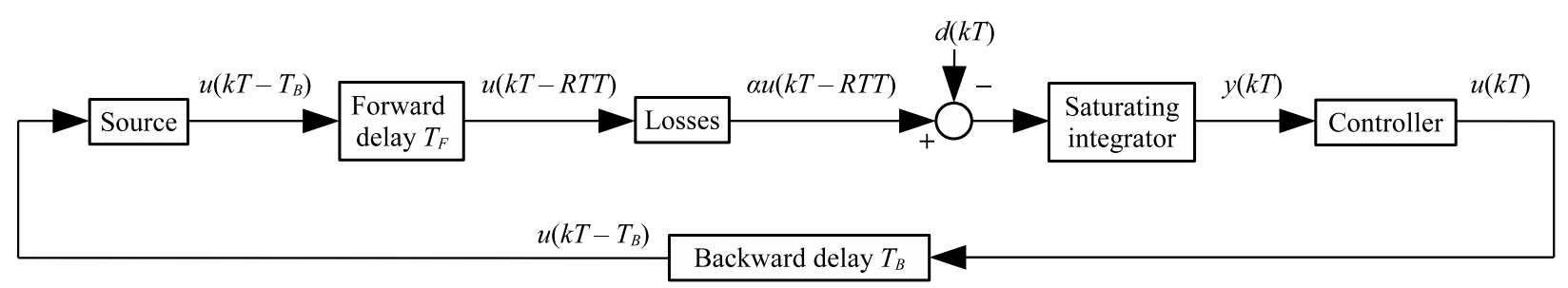

Fig. 1. Network model.

for $i=2, \ldots, n . \boldsymbol{A}$ is an $n \times n$ state matrix,

$$
\boldsymbol{A}=\left[\begin{array}{ccccc}
1 & \alpha & 0 & & 0 \\
0 & 0 & 1 & \cdots & 0 \\
& \vdots & & \ddots & \vdots \\
0 & 0 & 0 & \cdots & 1 \\
0 & 0 & 0 & & 0
\end{array}\right]
$$

and $\boldsymbol{b}, \boldsymbol{o}, \boldsymbol{q}$ are $n \times 1$ vectors,

$$
\boldsymbol{b}=\left[\begin{array}{c}
0 \\
0 \\
\vdots \\
0 \\
1
\end{array}\right], \quad \boldsymbol{o}=\left[\begin{array}{c}
-1 \\
0 \\
\vdots \\
0 \\
0
\end{array}\right], \quad \boldsymbol{q}=\left[\begin{array}{c}
1 \\
0 \\
\vdots \\
0 \\
0
\end{array}\right]
$$

The state space equation can also be written as follows:

$$
\left\{\begin{aligned}
x_{1}[(k+1) T] & =x_{1}(k T)+\alpha x_{2}(k T)-h(k T), \\
x_{2}[(k+1) T] & =x_{3}(k T), \\
& \vdots \\
x_{n-1}[(k+1) T] & =x_{n}(k T), \\
x_{n}[(k+1) T] & =u(k T) .
\end{aligned}\right.
$$

The desired state of the system is denoted by $\boldsymbol{x}_{\boldsymbol{d}}=\left[\begin{array}{llll}x_{d 1} & x_{d 2} & \cdots & x_{d n}\end{array}\right]^{T}$, where $x_{d 1}=y_{d}$ is the demand value of the queue length. It can be noticed from (8) that all other components of $\boldsymbol{x}_{\boldsymbol{d}}$ are equal to zero for $h(k T)=0$.

\section{Proposed control strategy}

In this section a control-theoretic approach is applied to design a discrete-time sliding-mode controller for the system considered. We begin with deriving the general form of a sliding-mode controller. The parameters of the sliding hyperplane are then chosen minimizing two different quality criteria. One involves the output of the system, and the other takes into account the whole state vector. Finally, the stability of the closed-loop system and its other important properties are proved.

3.1. Sliding-mode controller design. We introduce a sliding hyperplane described by the following equation:

$$
s(k T)=c^{T} \boldsymbol{e}(k T)=0,
$$

where $c^{T}=\left[\begin{array}{llll}c_{1} & c_{2} & \cdots & c_{n}\end{array}\right]$ is a vector satisfying $\boldsymbol{c}^{T} \boldsymbol{b} \neq 0$, and $\boldsymbol{e}(k T)=\boldsymbol{x}_{\boldsymbol{d}}-\boldsymbol{x}(k T)$ denotes the closedloop system error. Substituting (4) into $\boldsymbol{c}^{T} \boldsymbol{e}[(k+1) T]=$ 0 , the following feedback control law can be derived:

$$
u(k T)=\left(\boldsymbol{c}^{T} \boldsymbol{b}\right)^{-1} \boldsymbol{c}^{T}\left[\boldsymbol{x}_{\boldsymbol{d}}-\boldsymbol{A} \boldsymbol{x}(k T)\right] .
$$

Using (6), (7) and (8), we can present (10) as

$$
u(k T)=c_{n}^{-1}\left[c_{1} y_{d}-c_{1} x_{1}(k T)-\sum_{i=2}^{n} c_{i-1} x_{i}(k T)\right] \text {. }
$$

With the application of this control strategy, the state matrix of the closed-loop system has the form $\boldsymbol{A}_{\boldsymbol{c}}=$ $\left[\boldsymbol{I}_{\boldsymbol{n}}-\boldsymbol{b}\left(\boldsymbol{c}^{T} \boldsymbol{b}\right)^{-1} \boldsymbol{c}^{T}\right] \boldsymbol{A}$. The characteristic polynomial of $\boldsymbol{A}_{c}$ can be found as follows:

$$
\begin{aligned}
\operatorname{det}\left(z \boldsymbol{I}_{\boldsymbol{n}}-\boldsymbol{A}_{\boldsymbol{c}}\right)= & z^{n}+\frac{c_{n-1}-c_{n}}{c_{n}} z^{n-1}+\cdots \\
& +\frac{c_{2}-c_{3}}{c_{n}} z^{2}+\frac{\alpha c_{1}-c_{2}}{c_{n}} z .
\end{aligned}
$$

The properties of the closed-loop system will be determined by the choice of the sliding hyperplane parameters $c_{1}, c_{2}, \ldots, c_{n}$. In the remaining part of this subsection, two performance indices will be considered. For both of them appropriate selection of elements of vector $c$, minimizing the different cost functionals will be obtained.

Case 1. In optimization problems we often consider a performance index involving values of the control signal and the output error. In this case we seek for a slidingmode control $u_{\text {opt }}(k T)$ that minimizes the following cost functional:

$$
J_{1}(u)=\sum_{k=0}^{\infty}\left\{u^{2}(k T)+w\left[y_{d}-y(k T)\right]^{2}\right\},
$$

where $w$ is a positive weighting factor adjusting the influence of the control signal and the output variable on the functional. According to Kwakernaak and Sivan (1972), for the time-invariant discrete-time system (4) the optimal control $u_{\text {opt }}(k T)$ that minimizes the cost functional (13) can be presented as

$$
u_{\mathrm{opt}}(k T)=-\boldsymbol{g} \boldsymbol{x}(k T)+r,
$$


where

$$
\begin{gathered}
\boldsymbol{g}=\boldsymbol{b}^{*} \boldsymbol{K}\left(\boldsymbol{I}_{\boldsymbol{n}}+\boldsymbol{b}^{*} \boldsymbol{K}\right)^{-1} \boldsymbol{A} \\
r=\boldsymbol{G}_{\boldsymbol{c}}(1)^{-1} y_{d}=\left[\boldsymbol{q}^{T}\left(\boldsymbol{I}_{\boldsymbol{n}}-\boldsymbol{A}+\boldsymbol{b} \boldsymbol{g}\right)^{-1} \boldsymbol{b}\right]^{-1} y_{d},
\end{gathered}
$$

where $(\cdot)^{*}$ denotes the complex conjugate matrix transpose, $\boldsymbol{G}_{\boldsymbol{c}}(z)$ is the transfer function of the closed-loop system, and semipositive matrix $\boldsymbol{K}$ satisfies $\boldsymbol{K}^{*}=\boldsymbol{K}$ and is determined by the following Ricatti equation:

$$
\boldsymbol{K}=\boldsymbol{A}^{*} \boldsymbol{K}\left(\boldsymbol{I}_{\boldsymbol{n}}+\boldsymbol{b b}^{*} \boldsymbol{K}\right)^{-1} \boldsymbol{A}+w \boldsymbol{q} \boldsymbol{q}^{*} .
$$

Because all elements of $\boldsymbol{A}, \boldsymbol{b}$ and $\boldsymbol{q}$ are real numbers, the complex conjugate matrix transpose $(\cdot)^{*}$ is equivalent to the matrix transpose $(\cdot)^{T}$, and the elements of $\boldsymbol{K}$ are also real numbers. Therefore, condition $\boldsymbol{K}^{*}=\boldsymbol{K}$ implies that $\boldsymbol{K}$ is symmetric.

In the case of the network system considered in this paper, the Ricatti equation needs to be solved analytically for the system of an arbitrary order $n$. The method proposed here is similar to the one used by Ignaciuk and Bartoszewicz (2008). It involves iterative substitution of $\boldsymbol{K}$ into the right hand side of Eqn. 17) and comparing the result with its left-hand side, so that at each iteration the number of independent elements of $\boldsymbol{K}$ is reduced.

We begin with

$$
\boldsymbol{K}_{0}=\left[\begin{array}{ccccc}
k_{11} & k_{12} & k_{13} & & k_{1 n} \\
k_{12} & k_{22} & k_{23} & \cdots & k_{2 n} \\
k_{13} & k_{23} & k_{33} & & k_{3 n} \\
& \vdots & & \ddots & \vdots \\
k_{1 n} & k_{2 n} & k_{3 n} & & k_{n n}
\end{array}\right] .
$$

Because matrix $\boldsymbol{K}$ is symmetric, in the following equations, in order to save space, we will represent the elements positioned below the diagonal by '*'. After the first analytical iteration, we obtain the following form:

$$
\boldsymbol{K}_{1}=\left[\begin{array}{ccccc}
k_{11} & \alpha\left(k_{11}-w\right) & k_{13} & & k_{1 n} \\
* & \alpha^{2}\left(k_{11}-w\right) & k_{23} & \cdots & k_{2 n} \\
* & * & k_{33} & & k_{3 n} \\
& \vdots & & \ddots & \vdots \\
* & * & * & \cdots & k_{n n}
\end{array}\right] .
$$

The next step is substituting $\boldsymbol{K}_{1}$ given by (19) into (17) and comparing its left and right-hand sides. We arrive at the next form of $\boldsymbol{K}$, which expresses the values of more elements in terms of $k_{11}$,

$$
\begin{aligned}
& \boldsymbol{K}_{2} \\
& =\left[\begin{array}{ccccc}
k_{11} & \alpha\left(k_{11}-w\right) & \alpha\left(k_{11}-2 w\right) & & k_{1 n} \\
* & \alpha^{2}\left(k_{11}-w\right) & \alpha^{2}\left(k_{11}-2 w\right) & \ldots & k_{2 n} \\
* & * & \alpha^{2}\left(k_{11}-2 w\right) & & k_{3 n} \\
& \vdots & & \ddots & \vdots \\
* & * & * & \ldots & k_{n n}
\end{array}\right] .
\end{aligned}
$$

We repeat this procedure until all elements of $\boldsymbol{K}$ are expressed as functions of $k_{11}$, weighting factor $w$ and system order $n$,

$$
\begin{aligned}
& \boldsymbol{K} \\
& =\left[\begin{array}{cccc}
k_{11} & \alpha\left(k_{11}-w\right) & \cdots & \alpha\left(k_{11}-(n-1) w\right) \\
* & \alpha^{2}\left(k_{11}-w\right) & \cdots & \alpha^{2}\left(k_{11}-(n-1) w\right) \\
\vdots & \vdots & \ddots & \vdots \\
* & * & \cdots & \alpha^{2}\left(k_{11}-(n-1) w\right)
\end{array}\right] .
\end{aligned}
$$

In order to determine $k_{11}$, we substitute (21) into the righthand side of 17 and compare the first elements of the obtained matrices. This results in

$$
\alpha^{2} k_{11}^{2}-\alpha^{2} w(2 n-1) k_{11}+\alpha^{2} w^{2}\left(n^{2}-n\right)-w=0 .
$$

Equation (22) has the following roots:

$$
\begin{aligned}
& k_{11}^{\prime}=\frac{1}{2 \alpha}\left[\alpha(2 n-1) w+\sqrt{w\left(\alpha^{2} w+4\right)}\right] \\
& k_{11}^{\prime \prime}=\frac{1}{2 \alpha}\left[\alpha(2 n-1) w-\sqrt{w\left(\alpha^{2} w+4\right)}\right]
\end{aligned}
$$

The determinant of every principal minor of $\boldsymbol{K}$ is given by $\operatorname{det}\left(\boldsymbol{K}_{\boldsymbol{r}}\right)=\alpha^{2} w^{r-1}\left[k_{11}-(r-1) w\right]$, where $r$ is the dimension of the minor considered. Therefore, since $w \geq 0$, $\boldsymbol{K}$ will be semipositive definite if and only if the condition $k_{11} \geq(n-1) w$ is satisfied. Therefore, only $k_{11}^{\prime}$ guarantees that matrix $\boldsymbol{K}$ is semipositive definite. Having found $\boldsymbol{K}$, we derive vector $\boldsymbol{g}$ by substituting $k_{11}^{\prime}$ into (15) and obtain

$$
\boldsymbol{g}=\gamma_{1}\left[\begin{array}{lllll}
1 / \alpha & 1 & 1 & \cdots & 1
\end{array}\right]
$$

where

$$
\gamma_{1}=\frac{1}{2}\left[\alpha \sqrt{w\left(\alpha^{2} w+4\right)}-\alpha^{2} w\right]
$$

From (16) we obtain

$$
r=\frac{\gamma_{1} y_{d}}{\alpha}
$$

Finally, the optimal control law can be written as

$$
u_{\mathrm{opt}}(k T)=\gamma_{1}\left[\frac{y_{d}-y(k T)}{\alpha}-\sum_{i=2}^{n} x_{i}(k T)\right] \text {. }
$$

Case 2. We can also analyze the situation where instead of the output error the whole state error vector is taken into account. In this case we seek for $u_{\mathrm{opt}}(k T)$ that minimizes the following cost functional:

$$
J_{2}(u)=\sum_{k=0}^{\infty}\left[u^{2}(k T)+\boldsymbol{e}^{T}(k T) \boldsymbol{W} \boldsymbol{e}(k T)\right],
$$

where

$$
\boldsymbol{W}=\left[\begin{array}{cccc}
w_{1} & 0 & \cdots & 0 \\
0 & w_{2} & \cdots & 0 \\
\vdots & \vdots & \ddots & \vdots \\
0 & 0 & \cdots & w_{2}
\end{array}\right]
$$


with $w_{1}$ and $w_{2}$ being positive constants that adjust the influence of the queue length error and the amount of data under way error, respectively. The optimal control $u_{\text {opt }}(k T)$ can be found using (14), (15) and (16). Equation (17) needs to be modified to

$$
\boldsymbol{K}=\boldsymbol{A}^{T} \boldsymbol{K}\left(\boldsymbol{I}_{\boldsymbol{n}}+\boldsymbol{b} \boldsymbol{b}^{T} \boldsymbol{K}\right)^{-1} \boldsymbol{A}+\boldsymbol{W} .
$$

Solving (30) in the same way as before, we arrive at the following form of matrix $K$ :

$$
\boldsymbol{K}=\left[\begin{array}{cccc}
k_{11} & \alpha\left(k_{11}-w_{1}\right) & \cdots & M_{1} \\
* & \alpha^{2}\left(k_{11}-w_{1}\right)+w_{2} & \cdots & M_{2} \\
\vdots & \vdots & & \vdots \\
* & * & \ddots & M_{2} \\
* & * & \cdots & M_{3}
\end{array}\right]
$$

where

$$
\begin{aligned}
& M_{1}=\alpha\left[k_{11}-(n-1) w_{1}\right], \\
& M_{2}=\alpha^{2}\left[k_{11}-(n-1) w_{1}\right], \\
& M_{3}=\alpha^{2}\left[k_{11}-(n-1) w_{1}\right]+(n-1) w_{2},
\end{aligned}
$$

and $k_{11}$ is determined by

$$
\begin{aligned}
& \alpha^{2} k_{11}^{2}-k_{11} w_{1} \alpha^{2}(2 n-1) \\
& \quad+w_{1}(n-1)\left(\alpha^{2} w_{1} n-w_{2}\right)-w_{1}=0 .
\end{aligned}
$$

Equation (33) has two roots,

$$
\begin{aligned}
k_{11}^{\prime}= & \frac{1}{2 \alpha}\left[w_{1} \alpha(2 n-1)\right. \\
& \left.+\sqrt{w_{1}^{2} \alpha^{2}+4 w_{1} w_{2}(n-1)+4 w_{1}}\right], \\
k_{11}^{\prime \prime}= & \frac{1}{2 \alpha}\left[w_{1} \alpha(2 n-1)\right. \\
& \left.-\sqrt{w_{1}^{2} \alpha^{2}+4 w_{1} w_{2}(n-1)+4 w_{1}}\right] .
\end{aligned}
$$

Only $k_{11}^{\prime}$ guarantees that $\boldsymbol{K}$ is semipositive definite. Now we can obtain $\boldsymbol{g}$ by substituting $k_{11}^{\prime}$ into (15),

$$
\boldsymbol{g}=\gamma_{2}\left[\begin{array}{llll}
1 / \alpha & 1 & \cdots & 1
\end{array}\right]
$$

where

$$
\gamma_{2}=\frac{\alpha \sqrt{w_{1}\left(\alpha^{2} w_{1}+4\right)+4 w_{1} w_{2}(n-1)}-\alpha^{2} w_{1}}{2+2 w_{2}(n-1)} .
$$

Now we can derive $r$ from 16 :

$$
r=\frac{\gamma_{2} y_{d}}{\alpha}
$$

We conclude that the optimal control law for the second quality criterion is given by

$$
u_{\mathrm{opt}}(k T)=\gamma_{2}\left[\frac{y_{d}-y(k T)}{\alpha}-\sum_{i=2}^{n} x_{i}(k T)\right] .
$$

Remark 1. As $w \rightarrow 0$ for the first criterion, and as $w_{1} \rightarrow 0$ for the second one, the influence of the output error on the value of the functional diminishes, and gains of the controllers decrease to zero. As $w \rightarrow \infty$ for the first criterion, and as $w_{1} \rightarrow \infty$ for any finite $w_{2}$ in the second case, the impact of the control signal is negligible, and the output error is to be reduced to zero as quickly as possible, regardless of the value of the control signal. The controllers then become dead-beat schemes, and their gains approach unity. State variables $x_{2}, x_{3}, \ldots, x_{n}$ are the delayed values of the control signal. This means that, as $w_{2} \rightarrow \infty$ with finite $w_{1}$ for the second cost functional, the value of the control signal dominates the quality criterion and gain $\gamma_{2}$ drops to zero. The relation between $\gamma_{1}$ and $w$ for $\alpha=0.97$ is shown in Fig. 2, Relations between gain $\gamma_{2}$ and the weighting coefficients $w_{1}$ and $w_{2}$ for the second controller are depicted in Figs. 3 and 4 , respectively.

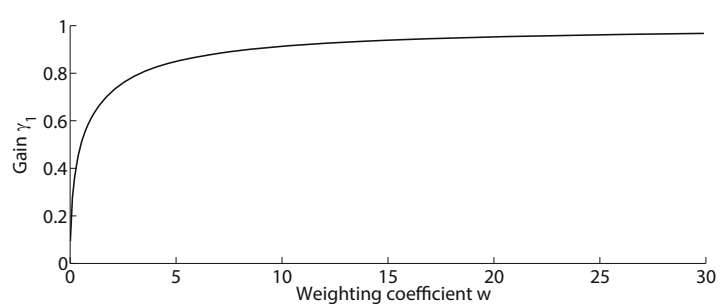

Fig. 2. Relation between $\gamma_{1}$ and $w$ for $\alpha=0.97$.

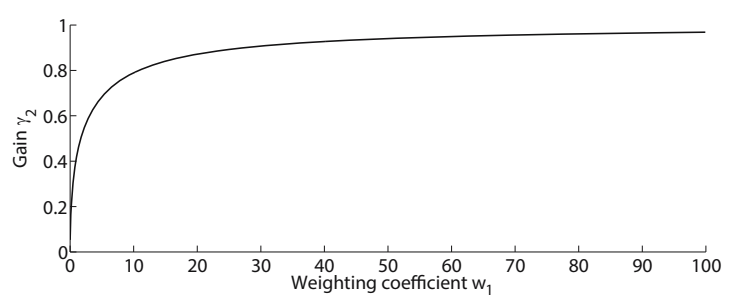

Fig. 3. Relation between $\gamma_{2}$ and $w_{1}$ for $w_{2}=0.25, n=9$ and $\alpha=0.97$.

3.2. Stability analysis. We notice that both quality criteria lead to $\boldsymbol{c}^{T}=\left[\begin{array}{lllll}\gamma / \alpha & \gamma & \cdots & \gamma & 1\end{array}\right]$, where $\gamma$ is either $\gamma_{1}$ or $\gamma_{2}$. Substituting this vector into (12), we obtain the characteristic polynomial

$$
\operatorname{det}\left(z \boldsymbol{I}_{\boldsymbol{n}}-\boldsymbol{A}_{\boldsymbol{c}}\right)=z^{n-1}[z-(1-\gamma)] .
$$




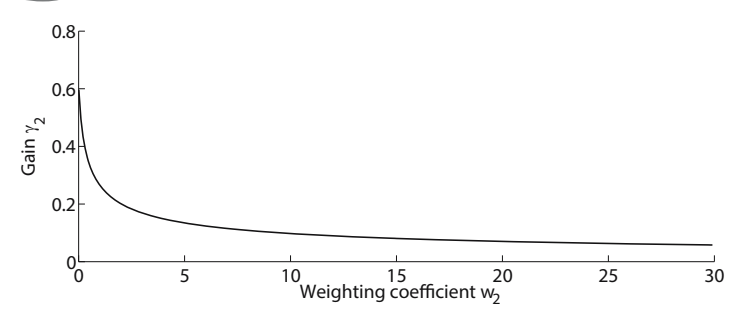

Fig. 4. Relation between $\gamma_{2}$ and $w_{2}$ for $w_{1}=1, n=9$ and $\alpha=0.97$.

A discrete-time closed-loop system is asymptotically stable if all the roots of the characteristic polynomial of its system state matrix are located inside the unit circle. The roots of (39) lie inside the unit circle if $\gamma \in(0,2)$. Since both $\gamma_{1}$ and $\gamma_{2}$ satisfy $\gamma \in(0,1)$, both of the proposed optimal controllers indeed guarantee the asymptotic stability of the system.

3.3. Properties of the proposed strategy. Properties of both optimal controllers will be formulated and proved simultaneously, again denoting by $\gamma$ both $\gamma_{1}$ and $\gamma_{2}$.

Theorem 1. If the proposed control strategy is applied, then the queue length never exceeds its demand value $y_{d}$.

Proof. Substituting (3) and (5) into (27) or (38), we obtain

$$
u_{o p t}(k T)=\gamma\left[\frac{y_{d}}{\alpha}-\sum_{i=0}^{k-1} u(i T)+\frac{1}{\alpha} \sum_{i=0}^{k-1} h(i T)\right]
$$

We assume that $y(m T) \leq y_{d}$ at some time instant $m \geq 0$. We will prove that this theorem is also true for $m+1$. The queue length at time $m+1$ can be expressed as

$$
\begin{aligned}
y[(m+1) T]= & y(m T)+\alpha u\left[\left(m-m_{\mathrm{RTT}}\right) T\right] \\
& -h(m T) .
\end{aligned}
$$

Using (3) and (40), we obtain

$$
\begin{aligned}
y[(m+1) T] & \\
= & y(m T)-h(m T) \\
& +\alpha \gamma\left[\frac{y_{d}}{\alpha}-\sum_{i=0}^{m-m_{\mathrm{RTT}}-1} u(i T)\right.
\end{aligned}
$$

$$
\begin{aligned}
& \left.+\frac{1}{\alpha} \sum_{i=0}^{m-m_{\mathrm{RTT}}-1} h(i T)\right] \\
= & \gamma y_{d}+y(m T)-\gamma \sum_{i=m-m_{\mathrm{RTT}}}^{m-1} h(i T)-h(m T) \\
& -\gamma\left[\alpha \sum_{i=0}^{m-m_{\mathrm{RTT}}-1} u(i T)-\sum_{i=0}^{m-1} h(i T)\right] \\
= & y_{d}-(1-\gamma)\left[y_{d}-y(m T)\right] \\
& -\gamma \sum_{i=m-m_{\mathrm{RTT}}}^{m-1} h(i T)-h(m T) .
\end{aligned}
$$

Since $\gamma \in(0,1)$ and $h(k T)$ is always nonnegative, $y[(m+1) T] \leq y_{d}$. Because $y(0) \leq y_{d}$, we conclude that $y(k T) \leq y_{d}$ for any $k \geq 0$. This ends the proof.

We notice from (8) that, if $x_{1}[(k+1) T]>0$, then the available bandwidth $d(k T)$ is fully used. The next theorem shows how to choose $y_{d}$ in order to ensure that the queue length is strictly positive.

Theorem 2. If the desired queue length

$$
y_{d}>d_{\max }\left(m_{\mathrm{RTT}}+1 / \gamma\right),
$$

then for any $k \geq m_{\mathrm{RTT}}+1$ the queue length is greater than zero.

Proof. From (3) it follows that $y(k T)=0$ for $k<m_{\mathrm{RTT}}+1$. Furthermore, we notice that both 27) and (38) lead to $u(0)=\gamma y_{d} / \alpha$, with $\gamma$ denoting $\gamma_{1}$ or $\gamma_{2}$, respectively. Using the above observations and (43) with (41), we notice that

$$
\begin{aligned}
& y\left[\left(m_{\mathrm{RTT}}+1\right) T\right] \\
& \quad=\alpha u(0)-h\left(m_{\mathrm{RTT}} T\right) \\
& \quad>d_{\max }\left(\gamma m_{\mathrm{RTT}}+1\right)-d_{\max }>0 .
\end{aligned}
$$

Now we shall demonstrate that the condition $y(m T)>0$ implies $y[(m+1) T]>0$. From (42) and 43], we obtain

$$
\begin{aligned}
& y[(m+1) T] \\
& =\gamma y_{d}+(1-\gamma) y(m T) \\
& \quad-\gamma \sum_{i=m-m_{\mathrm{RTT}}}^{m-1} h(i T)-h(m T) \\
& \geq \gamma y_{d}-\gamma m_{\mathrm{RTT}} d_{\max }-d_{\max } \\
& =\gamma\left[y_{d}-d_{\max }\left(m_{\mathrm{RTT}}+\frac{1}{\gamma}\right)\right]>0 .
\end{aligned}
$$

It follows from (44) and (45) that, indeed, if (43) holds, then $y(k T)>0$ for $k \geq m_{\mathrm{RTT}}+1$. This completes the induction proof. 
In order to be applied in a real network, any flow control algorithm should generate transmission rates that are always nonnegative and limited by some predictable, finite value. This property is demonstrated in the next theorem.

Theorem 3. With the application of the proposed controller, data transmission rates are always nonnegative and upper bounded, i.e., for any $k \geq 0$,

$$
0 \leq u(k T) \leq \max \left(\gamma y_{d} / \alpha, d_{\max } / \alpha\right)
$$

Proof. Let us assume that (46) holds for some $m \geq 0$. We shall prove that the proposition is true also for $m+1$. Using (40), we get

$$
\begin{aligned}
u[(m+1) T] & {\left[\frac{y_{d}}{\alpha}-\sum_{i=0}^{m} u(i T)+\frac{1}{\alpha} \sum_{i=0}^{m} h(i T)\right] } \\
=\gamma & {\left[\frac{y_{d}}{\alpha}-\sum_{i=0}^{m-1} u(i T)+\frac{1}{\alpha} \sum_{i=0}^{m-1} h(i T)\right] } \\
& -\gamma\left[u(m T)-\frac{1}{\alpha} h(m T)\right] \\
= & (1-\gamma) u(m T)+\frac{\gamma}{\alpha} h(m T) .
\end{aligned}
$$

From (40) we have $u(0)=\gamma y_{d} / \alpha$. We conclude that (46) indeed holds for any $k \geq 0$.

Let us finally notice that, if the source is not persistent or if the round trip time is not known exactly, then a similar approach as the one proposed by Pietrabissa et al. (2006) can be adopted. In that paper an adaptive controller combining the advantages of control-theoretic and fuzzy-logic approaches was proposed to address the issue of source saturation as well as uncertain, possibly timevarying transmission delays. Furthermore, if the round trip time is not known exactly, and in particular when it is not a multiple of the discretisation period, then our optimal controller can be equipped with a saturating element and a similar approach to the one proposed by Bartoszewicz (2006) can also be effectively applied. However, when the round trip time is not known exactly, then conditions stated in Theorems 1 and 2 become more restrictive.

\section{Simulation results}

To verify the properties of the proposed control strategy, computer simulations have been performed. The sampling time $T$ was selected as $1 \mathrm{~ms}$. The round trip time $R T T$ in the virtual circuit was assumed to be $8 \mathrm{~ms}$. From this follows $m_{\mathrm{RTT}}=8$ and $n=9$. The maximum available bandwidth $d_{\max }=6 \mathrm{~kb}$. The bandwidth actually available for the data transfer is shown in Fig. 5. Sudden changes of large amplitude occur in the function $d$,

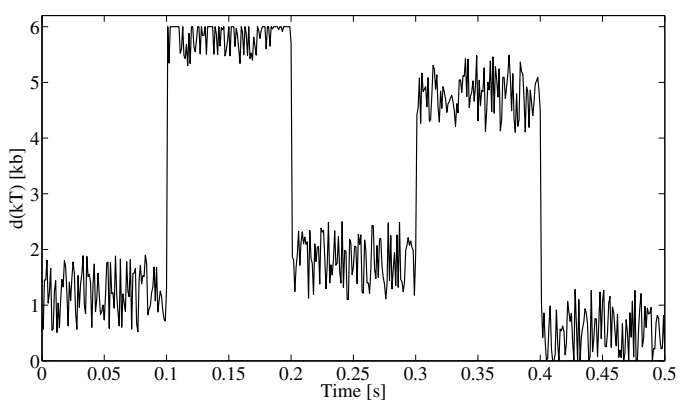

Fig. 5. Available bandwidth.

Table 1. Parameters of the first controller.

\begin{tabular}{|c|c|c|c|}
\hline$w$ & $\gamma_{1}$ & $y_{d}^{\prime}[\mathrm{kb}]$ & $y_{d}[\mathrm{~kb}]$ \\
\hline \hline 10 & 0.9117 & 54.6 & 56 \\
\hline 0.5 & 0.4899 & 60.3 & 62 \\
\hline
\end{tabular}

which reflects the most difficult possible conditions in the network. It is assumed that $3 \%$ of data is lost during the transmission, which corresponds to $\alpha=0.97$.

The derived parameters for the first controller: the gain $\gamma_{1}$ obtained from (25), the minimum demand queue length $y_{d}^{\prime}$ calculated from the condition (43), and the queue length actually used in the simulation $y_{d}$ are shown in Table 11. The results of the simulations are shown in Figs. 6 and 7 . The value of the control signal at the beginning of the transmission process is shown in Fig. 8

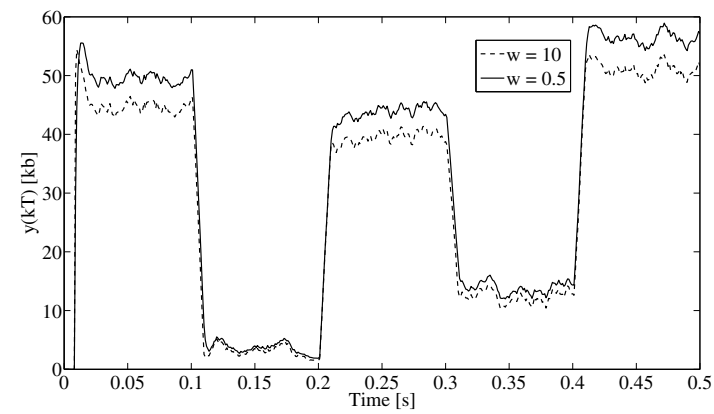

Fig. 6. Buffer occupancy with the first controller for different values of $w$.

Similar tests were performed with the second controller. The weighting factor $w_{2}$ was selected as 5 and the resulting parameters of the second controller are shown in Table 2. The gain $\gamma_{2}$ was derived from (36), and $y_{d}^{\prime}$ from (43). Simulation results are shown in Figs. 9 and 10 , Again, the value of the control signal at the beginning of the transmission process is shown more clearly in Fig. 11.

It can be seen from the figures that the transmission rates calculated by both the algorithms are always nonnegative and upper bounded. Furthermore, the queue length 


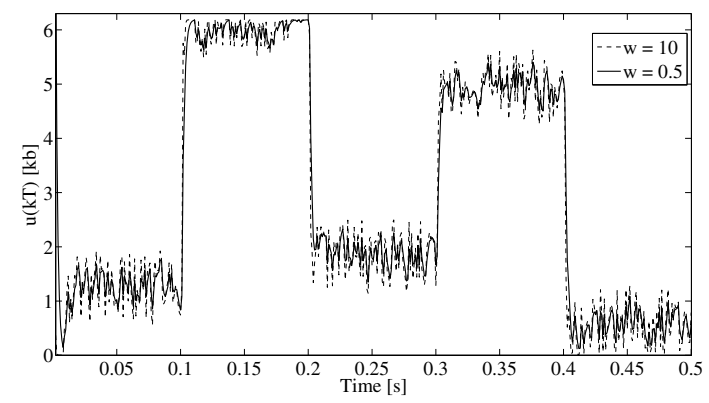

Fig. 7. Transmission rates with the first controller for different values of $w$.

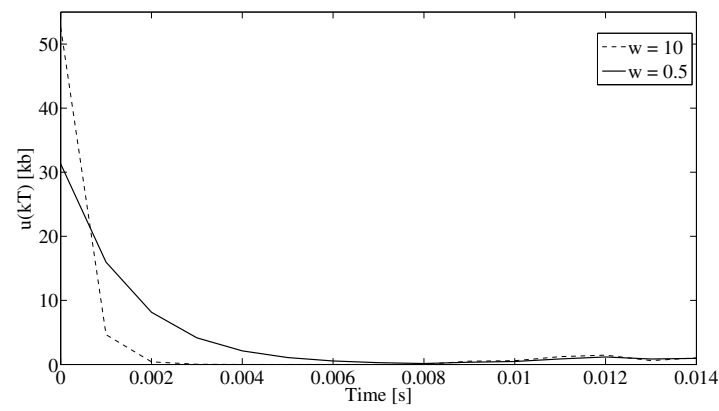

Fig. 8. Transmission rates with the first controller at the beginning of transmission.

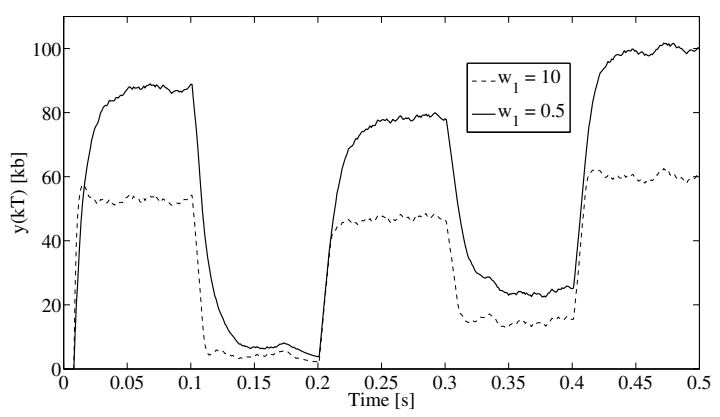

Fig. 9. Buffer occupancy with the second controller for different values of $w_{1}$

never exceeds its demand value, and for $k \geq m_{\mathrm{RTT}}+1$ it never decreases to zero. This means that there is no risk of data loss resulting from buffer overflow, and that all of the available bandwidth is used. Consequently, maximum throughput possible in the network is achieved.

Table 2. Parameters of the second controller.

\begin{tabular}{|c|c|c|c|}
\hline$w_{1}$ & $\gamma_{2}$ & $y_{d}^{\prime}[\mathrm{kb}]$ & $y_{d}[\mathrm{~kb}]$ \\
\hline \hline 10 & 0.3779 & 63.9 & 66 \\
\hline 0.5 & 0.1015 & 107.1 & 110 \\
\hline
\end{tabular}

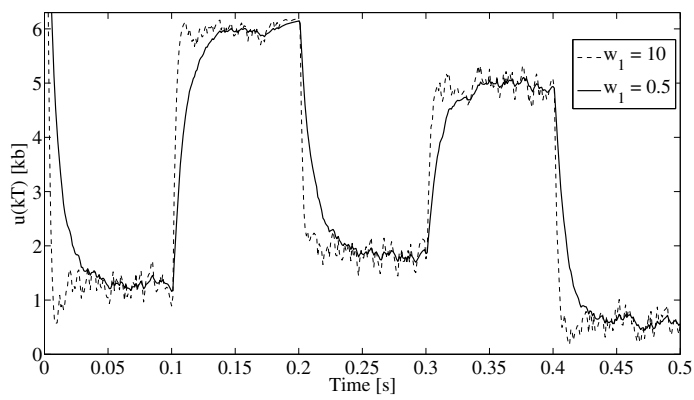

Fig. 10. Transmission rates with the second controller for different values of $w_{1}$.

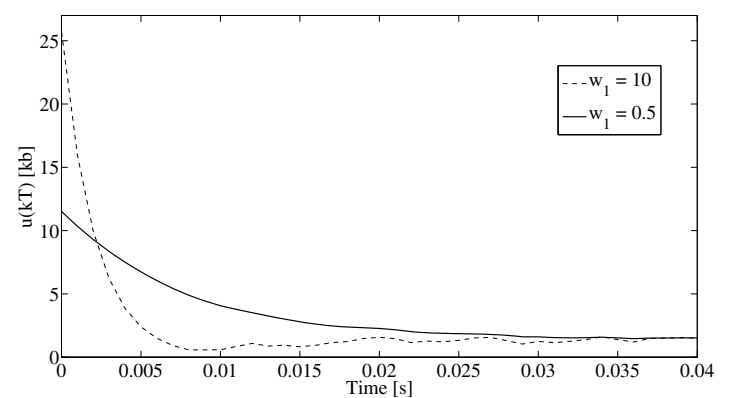

Fig. 11. Transmission rates with the second controller at the beginning of transmission.

Both algorithms can be adjusted to specific requirements using the appropriate weighting factors. As could be expected, changes in $w$ of the first criterion have a similar impact as changes of $w_{1}$ for the second case. Larger values of $w$ and $w_{1}$ result in faster tracking of the output flow. This, in turn, allows allocating smaller buffers, while still utilizing the full available bandwidth. On the other hand, smaller $w$ and $w_{1}$ lead to smaller values of the control signal at the beginning of the transmission process. This also makes the control signal smoother, which is advantageous for transmission consistency. Changes in $w_{2}$ for the second case have the opposite effect, as has already been stated in the previous section.

In all the above simulations the main goal was to fully utilize the available bandwidth. Therefore, the demand queue length was selected according to Theorem 2 . However, the condition (43) may not be satisfied for two reasons. Firstly, there may be not enough physical memory in the congested node. Secondly, we can deliberately lower $y_{d}$ in order to reduce jitter and latency, and in this way improve the Quality of Service (QoS) in the network. Therefore, for the last simulation scenario we consider the case of $w_{1}=10, w_{2}=5$ for the second functional, but now we choose $y_{d}=58<y_{d}^{\prime}$. The results are presented in Figs. 12 and 13. The unused bandwidth, which is equal to $d(k T)-h(k T)$, is presented in Fig. 14 As can be ob- 
served, the proposed control algorithm ensures fairly good bandwidth utilization even with a lowered demand queue length.

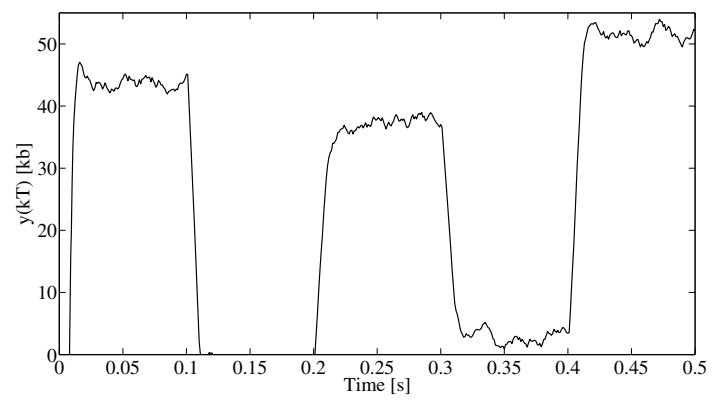

Fig. 12. Buffer occupancy for lowered $y_{d}$.

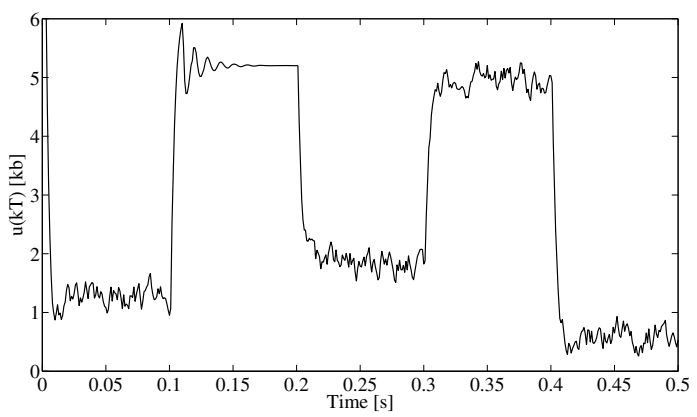

Fig. 13. Transmission rate for lowered $y_{d}$.

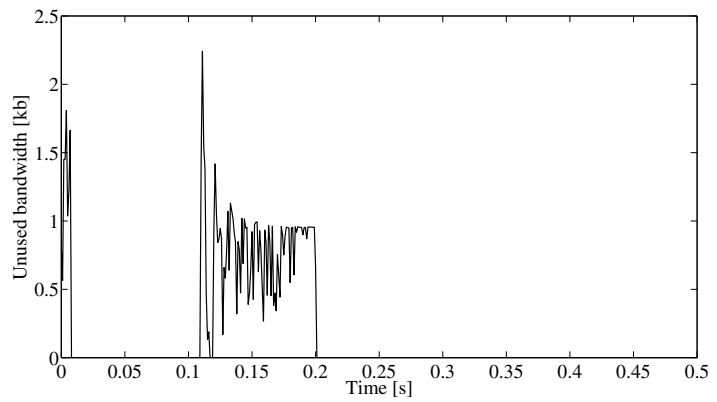

Fig. 14. Unused bandwidth for lowered $y_{d}$.

\section{Conclusion}

In this paper, two LQ optimal sliding-mode flow controllers for a single virtual circuit in data transmission networks were proposed. Possible data losses during the transmission were explicitly taken into account. The design procedure was based on minimization of two different quadratic cost functionals, and solving the resulting matrix Ricatti equation. The closed-loop system stability was demonstrated. The condition for full bandwidth consumption was formulated and proved. It was also proved that the rates generated by the controller are always nonnegative and upper bounded. Finally, it is worth pointing out that the results presented by Ignaciuk and Bartoszewicz (2008) may be regarded as a special case of the more general analysis performed in this paper.

\section{Acknowledgment}

This work was performed in the framework of the project Optimal sliding mode control of time delay systems financed by the National Science Centre (DEC 2011/01/B/ST7/02582). The kind support provided by the Foundation for Polish Science under a Mistrz grant is also acknowledged.

This work is an extended version of the paper Linear quadratic optimal sliding-mode controller for connectionoriented communication networks with lossy links presented at the 11th International Conference on Diagnostics of Processes and Systems (Łagów Lubuski, Poland, 2013).

\section{References}

Bartoszewicz, A. (2006). Nonlinear flow control strategies for connection-oriented communication networks, IEE Proceedings on Control Theory and Applications (Part D) 153(1): 21-28.

Bartoszewicz, A. and Zuk, J. (2009). Discrete time sliding mode flow controller for multi-source single-bottleneck connection-oriented communication networks, Journal of Vibration and Control 15(11): 1745-1760.

Drazenovic, B. (1969). The invariance conditions in variable structure systems, Automatica 5(3): 287-295.

Edwards, C., Alwi, H. and Tan, C.P. (2012). Sliding mode methods for fault detection and fault tolerant control with application to aerospace systems, International Journal of Applied Mathematics and Computer Science 22(1): 109-124, DOI: 10.2478/v10006-012-0008-7.

Edwards, C. and Spurgeon, S. (1994). On the development of discontinuous observers, International Journal of Control 59(4): 1211-1229.

Emelyanov, S.V. (1967). Variable Structure Control System, Nauka, Moscow.

Floquet, T., Edwards, C. and Spurgeon, S. (2007). On sliding mode observers for systems with unknown inputs, International Journal of Adaptive Control and Signal Processing 21(8-9): 638-656.

Fridman, L., Levant, A. and Davila, J. (2007). Observation of linear systems with unknown inputs via high-order sliding-modes, International Journal of Systems Science 38(10): 773-791.

Gao, W., Wang, Y. and Homaifa, A. (1995). Discrete-time variable structure control systems, IEEE Transactions on Industrial Electronics 42(2): 117-122. 
Haskara, I., Ozguner, U. and Utkin, V. (1998). On sliding mode observers via equivalent control approach, International Journal of Control 71(6): 1051-1067.

Hung, J.Y., Gao, W. and Hung, J.C. (1993). Variable structure control: A survey, IEEE Transactions on Industrial Electronics 40(1): 2-22.

Ignaciuk, P. and Bartoszewicz, A. (2008). Linear quadratic optimal discrete-time sliding-mode controller for connectionoriented communication networks, IEEE Transactions on Industrial Electronics 55(11): 4013-4021.

Ignaciuk, P. and Bartoszewicz, A. (2009). Linear quadratic optimal sliding mode flow control for connection-oriented communication networks, International Journal of Robust and Nonlinear Control 19(4): 442-461.

Ignaciuk, P. and Bartoszewicz, A. (2011). Discrete-time slidingmode congestion control in multisource communication networks with time-varying delay, IEEE Transactions on Control Systems Technology 19(4): 852-867.

Ignaciuk, P. and Bartoszewicz, A. (2013). Congestion Control in Data Transmission Networks: Sliding Mode and Other Designs, Springer-Verlag, London.

Jin, J., Wang, W. and Palaniswami, M. (2009). A simple framework of utility max-min flow control using sliding mode approach, IEEE Communications Letters 13(5): 360-362.

Jing, Y., He, L., Dimirovski, G. and Zhu, H. (2007). Robust stabilization of state and input delay for active queue management algorithm, Proceedings of the American Control Conference, New York, NY, USA, pp. 3083-3087.

Jing, Y., Yu, N., Kong, Z. and Dimirovski, G. (2008). Active queue management algorithm based on fuzzy sliding model controller, Proceedings of the 17th IFAC World Congress, Seoul, Korea, pp. 6148-6153.

Kwakernaak, H. and Sivan, R. (1972). Linear Optimal Control Systems, Wiley-Interscience, New York, NY.

Levant, A. (1993). Sliding order and sliding accuracy in sliding mode control, International Journal of Control 58(6): $1247-1263$.

Mnasri, C. and Gasmi, M. (2011). LMI-based adaptive fuzzy integral sliding mode control of mismatched uncertain systems, International Journal of Applied Mathematics and Computer Science 21(4): 605-615, DOI: 10.2478/v10006011-0047-5.

Pietrabissa, A., Priscoli, F. D., Fiaschetti, A. and Paolo, F. D. (2006). A robust adaptive congestion control for communication networks with time varying delays, Proceedings of the IEEE International Conference on Control Applications, Munich, Germany, pp. 2093-2098.
Sira-Ramirez, H. (1993). On the dynamical sliding mode control of nonlinear systems, International Journal of Control 57(5): 1039-1061.

Tomera, M. (2010). Nonlinear controller design of a ship autopilot, International Journal of Applied Mathematics and Computer Science 20(2): 271-280, DOI: 10.2478/v10006010-0020-8.

Utkin, V. (1977). Variable structure systems with sliding modes, IEEE Transactions on Automatic Control 22(2): 212-222.

Utkin, V. and Shi, J. (1996). Integral sliding mode in systems operating under uncertainty conditions, 35th IEEE Conference on Decision and Control, Kobe, Japan, pp. 45914596.

Veluvolu, K.C. and Soh, Y.C. (2009). Discrete-time slidingmode state and unknown input estimations for nonlinear systems, IEEE Transactions on Industrial Electronics 56(9): 3443-3345.

Yan, M., Kolemisevska-Gugulovska, T., Jing, Y. and Dimirovski, G. (2007). Robust discrete-time sliding-mode control algorithm for TCP networks congestion control, Proceedings of TELSIKS, Niš, Serbia, pp. 393-396.

Yang, M., Jing, Y., Dimirovski, G. and Zhang, N. (2007). Stability and performance analysis of a congestion control algorithm for networks, Proceedings of the 46th IEEE Conference on Decision and Control, New Orleans, LA, USA pp. 4453-4458.

Zhang, N., Yang, M., Jing, Y. and Zhang, S. (2009). Congestion control for DiffServ network using second-order sliding mode control, IEEE Transactions on Industrial Electronics 56(9): 3330-3336.

Zheng, X., Zhang, N., Dimirovski, G. and Jing, Y. (2008). Adaptive sliding mode congestion control for DiffServ network, Proceedings of the 17th IFAC World Congress, Seoul, Korea, pp. 12983-12987.

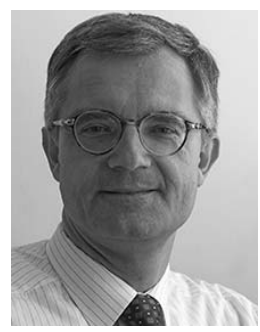

Andrzej Bartoszewicz received the M.Sc. degree in 1987 and the Ph.D. degree in 1993, both from the Łódź University of Technology, Poland. Then he obtained the postdoctoral degree in control engineering and robotics from the Academy of Mining and Metallurgy in Cracow, Poland. He was a visiting scholar at Purdue University, West Lafayette, USA, and at Strathclyde University, Glasgow, UK. Then for one year he was at the University of Leicester, UK. Currently he is a professor at the Technical University of Łódź, a vice dean of the Faculty of Electrical, Electronic, Computer and Control Engineering, the head of the Electric Drive and Industrial Automation Group and a vicedirector of the Institute of Automatic Control. He has published three monographs and over 250 papers, primarily in the fields of sliding mode control and congestion control in data transmission networks. 


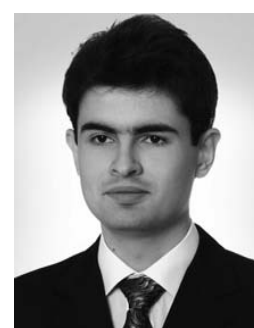

Piotr Leśniewski received the Master's degree in control engineering and robotics from the Łódź University of Technology in 2012. Currently he is a Ph.D. student at that university. His main research interests include discrete time sliding mode control and time-delay systems.

Received: 17 April 2013

Revised: 4 August 2013 